\title{
Delayed cerebral infarction due to cerebral venous air emboli after cardiac arrest
}

Yoon-Kyung Lee, MD'; Jin-Heon Jeong, MD ${ }^{1,2}$

'Department of Neurology, Stroke Center, Dong-A University Hospital, Busan, Republic of Korea ${ }^{2}$ Department of Intensive Care Medicine, Dong-A University Hospital, Busan, Republic of Korea

\section{Journal of Neurocritical} Care

\section{IMAGES IN \\ NEUROCRITICAL CARE}

Received: May 12, 2021

Revised: June 6, 2021

Accepted: June 8, 2021

Corresponding Author:

Jin-Heon Jeong, MD

Department of Intensive Care Medicine and Department of Neurology, Stroke Center, Dong-A University Hospital, Dong-A University College of Medicine, 26 Daesingongwon-ro, Seo-gu, Busan 49201, Korea

Tel: +82-51-240-5266

Fax: +82-51-244-8338

E-mail: jhjeong@dau.ac.kr
A 63-year-old woman had a sudden cardiac arrest while undergoing tooth scaling. After 4 minutes, the return of spontaneous circulation (ROSC) was achieved. She was stuporose, but other neurological examinations were within normal limits. The patient had a history of meningitis, and she had undergone ventriculoperitoneal shunt surgery for hydrocephalus in her 20s. Brain computed tomography performed immediately after ROSC showed cerebral air emboli (Fig. 1A). Brain magnetic resonance imaging (MRI) after 5 hours showed no acute lesions (Fig. 1B). Targeted temperature management was performed. Her consciousness improved to a drowsy state, but left hemiparesis was observed. Brain MRI performed on the 6th day showed gyriform infarction in both frontoparietal cortices that were consistent with air emboli (Fig. 1C-E).

Cerebral air embolism can lead to arterial and venous infarctions $[1,2]$. They can lead to blood flow obstruction and induce an inflammatory reaction with the breakdown of the blood-brain barrier [3]. In this case, the delayed cerebral infarction was detected by follow-up brain imaging. Cerebral venous air emboli can cause delayed cerebral infarction because the collateral circulation in the cerebral venous system allows for compensation. Even if the initial MRI is negative, repeated brain MRI may help detect cerebral infarctions caused by cerebral venous air emboli.

\section{ARTICLE INFORMATION}

\section{Ethics statement}

This case was reviewed and approved by the Institutional Review Board of Dong-A University Hospital (IRB No. DAUHIRB-21 -105). Informed consent was waived by the Board.

\section{Conflict of interest}

Dr. Jeong JH is an editorial board member of the journal but was not involved in the peer reviewer selection, evaluation, or decision process of this article. No other potential conflicts of interest relevant to this article were reported.

\section{Funding}

This research was supported by Basic Science Research Program through the National Research Foundation of Korea (NRF) funded by the Ministry of Science, ICT \& Future Planning (grant number: 2018R1C1B5086622). 

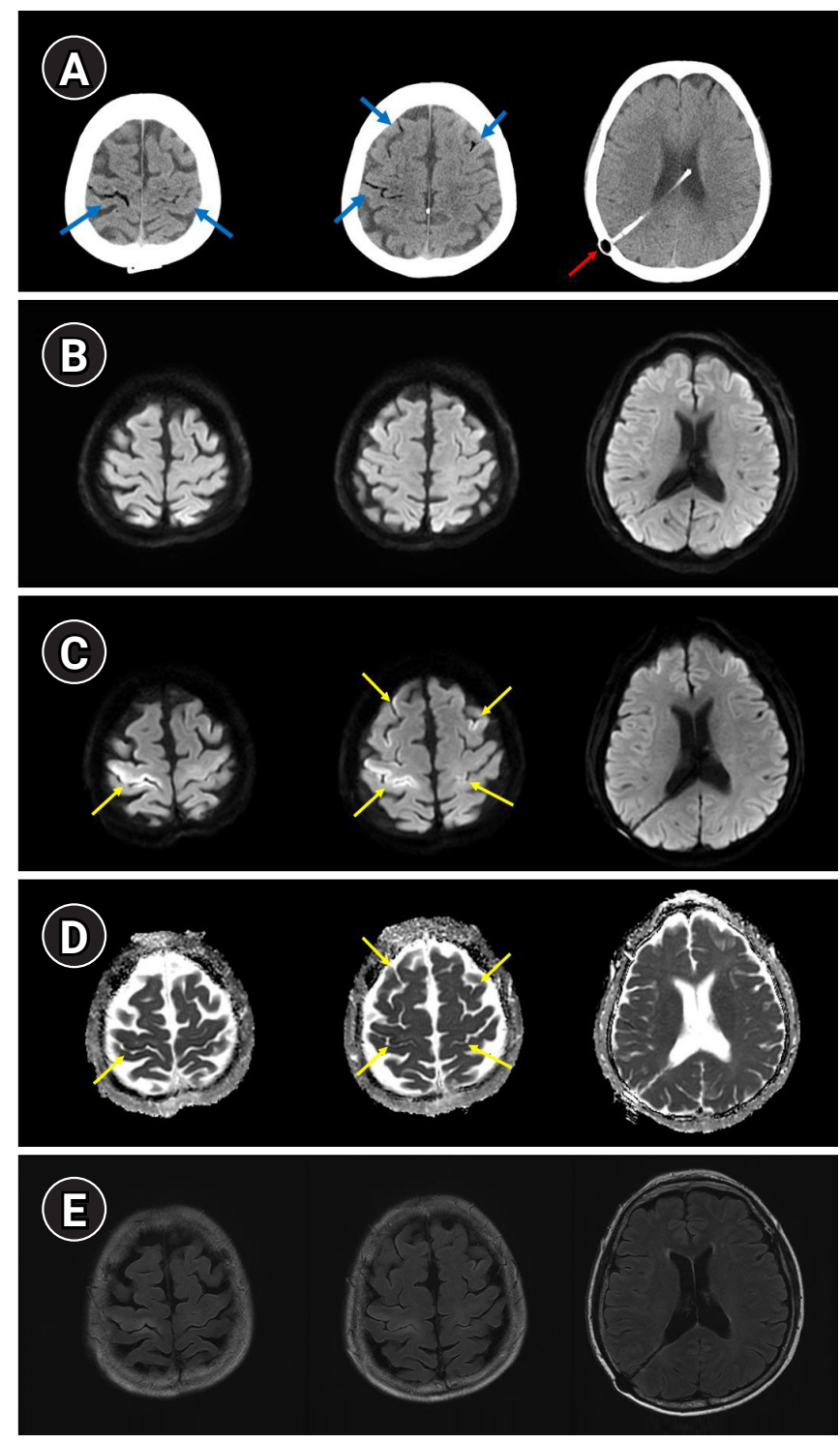

Fig. 1. Brain computed tomography and magnetic resonance imaging (MRI). (A) Non-contrast computed tomography of the head performed immediately after the return of spontaneous circulation (ROSC) showing air bubbles in the frontoparietal sulci (blue arrow). A ventriculoperitoneal shunt catheter was inserted in the right lateral ventricle (red arrow). (B) Diffusion-weighted brain MRI 5 hours after ROSC showing no acute lesions. (C-E) A follow-up brain MRI was performed on the 6th day after ROSC. Diffusion-weighted images and apparent diffusion coefficient images showed focal gyriform infarction in both frontoparietal cortices, especially in the right motor cortex (yellow arrows). Fluid-attenuated inversion recovery images showed high signal intensities in the same area.

\section{ORCID}

Yoon-Kyung Lee Jin-Heon Jeong

https://orcid.org/0000-0003-2602-6623

https://orcid.org/0000-0002-5878-9206

\section{Author contributions}

Conceptualization: JHJ. Data curation: all authors. Formal analysis: all authors. Funding acquisition: JHJ. Methodology: all authors. Project administration: all authors. Visualization: all authors. Writing-original draft: all authors. Writing-review \& editing: all authors.

\section{REFERENCES}

1. Jeon SB, Kim JS, Lee DK, Kang DW, Kwon SU. Clinicoradiological characteristics of cerebral air embolism. Cerebrovasc Dis 2007;23:459-62.

2. Kim YJ, Jeon SB. Cerebral air embolism treated using hyperbaric oxygen therapy.J Neurocrit Care 2019;12:64-65.

3. Lai D, Jovin TG, Jadhav AP. Cortical vein air emboli with gyriform infarcts. JAMA Neurol 2013;70:939-40. 